\title{
Introduction : Les mutations de la famille agricole
}

Statut économique • Pluriactivité • Revenus

France Caillavet, François Facchini et Catherine Moreddu

\section{(2) OpenEdition \\ 1 Journals}

Édition électronique

URL : http://journals.openedition.org/economierurale/86

DOI : $10.4000 /$ economierurale.86

ISSN : 2105-2581

Éditeur

Société Française d'Économie Rurale (SFER)

Édition imprimée

Date de publication : 30 décembre 2005

Pagination : 7-9

ISSN : 0013-0559

\section{Référence électronique}

France Caillavet, François Facchini et Catherine Moreddu, «Introduction : Les mutations de la famille agricole ", Économie rurale [En ligne], 289-290 | Septembre-décembre 2005, mis en ligne le 02 mars 2009, consulté le 20 avril 2019. URL : http://journals.openedition.org/economierurale/86 ; DOI :

$10.4000 /$ economierurale.86

Ce document a été généré automatiquement le 20 avril 2019.

(c) Tous droits réservés 


\title{
Introduction : Les mutations de la famille agricole
}

\author{
Statut économique $\bullet$ Pluriactivité $・$ Revenus \\ France Caillavet, François Facchini et Catherine Moreddu
}

1 La place de la femme en agriculture a suivi les évolutions sociales. La famille agricole a connu en ce sens des mutations équivalentes à celles de la société dans son ensemble, même si comme le rappelle Hubert Bosse-Platière, il est notoirement connu que l'on divorce moins chez les agriculteurs, et que l'on a moins qu'ailleurs des enfants hors mariage. La particularité de la famille agricole se trouve selon Alice Barthez dans la réunion au sein d'une même structure de la famille et de la profession. C'est ce qui a d'ailleurs inspiré les nombreuses lois d'orientation agricole en France qui se sont généralement données pour objectif de protéger l'exploitation familiale. C'est dans ce cadre social et législatif qu'il faut comprendre l'évolution du rôle de la femme sur l'exploitation. La femme est progressivement passée du statut d'épouse, à ceux d'aide familiale, d'associée et de chef d'exploitation. Dans leur article respectif, c'est ce que nous montrent Hubert Bosse-Platière et Alice Barthez à travers l'évolution du droit et l'observation des rapports quasi salariaux à l'intérieur de la famille agricole. Ils analysent l'évolution du droit par les changements de comportement et celle des mœurs par le désir d'émancipation des femmes. Ce désir éclaire les nouveaux rapports familiaux, car il conduit les femmes à travailler hors de l'exploitation et à participer à la prise de décision, et les hommes à s'installer avant d'être marié, s'éloignant ainsi du modèle traditionnel. Ils expliquent aussi l'apparition de nouveaux statuts comme celui d'agricultrice coexploitante et, plus généralement, l'évolution du droit rural. La relation entre le nouveau rôle de la femme en agriculture et la politique publique apparaît ainsi naturelle, même si parfois le droit est inadapté aux comportements réels des acteurs (en retard ou en avance).

2 La mesure des revenus agricoles est un problème dont l'analyse peut sembler indépendante de la question des mutations de la famille agricole et de la place de la femme en agriculture. Or la principale source de pluriactivité provient de l'activité féminine hors exploitation. Ainsi la volonté d'émancipation de la femme s'est trouvée 
fortement relayée par la nécessité économique de diversifier les sources de revenu du ménage agricole, provoquant en retour une transformation des rapports de travail dans l'exploitation. Quatre articles, issus de deux ateliers consacrés à la pluriactivité et aux revenus des ménages agricoles, ont été retenus pour illustrer cette problématique.

L'article de Jill Findeis, Hema Swaminathan et Anuja Jayaraman examine, à l'aide de données d'enquêtes menées aux États-Unis, la contribution des femmes agricultrices à l'emploi agricole et non agricole et leur rôle dans la gestion de l'exploitation. Des modèles de négociation sont utilisés à cette occasion pour identifier les facteurs qui déterminent les choix en matière d'emploi et de gestion. L'étude empirique montre qu'au cours des deux dernières décennies, la part des femmes agricultrices ayant une activité non agricole a nettement augmenté, ce qui a entraîné aux États-Unis une hausse des revenus des ménages agricoles, une amélioration de l'accès aux avantages du salariat et une convergence entre les revenus moyens des ménages agricoles et ceux des autres ménages. L'origine de la transmission de l'exploitation, les capacités humaines de l'agricultrice et son stade dans le cycle de vie ont une grande influence sur l'arbitrage des agricultrices en matière d'emploi. Si l'exploitation vient de sa famille, l'agricultrice se considère comme davantage impliquée dans sa gestion. Ainsi, même si elle travaille à l'extérieur, ce qui est souvent le cas, elle est davantage susceptible de pratiquer la pluriactivité sur et en dehors de l'exploitation. Par ailleurs, lorsque le conjoint/compagnon travaille à temps partiel en dehors de l'exploitation, l'âge de la femme semble un facteur plus important, la pluriactivité suivant le cycle de vie traditionnel. Enfin, la participation de la femme agricultrice à la prise de décision est renforcée si l'exploitation vient de sa famille alors qu'elle s'amenuise si l'exploitation vient de la famille de son conjoint/compagnon.

4 L'article de Berkeley Hill, présenté en séance plénière, rappelle sur la base d'une bibliographie bien documentée que les agriculteurs sont propriétaires d'un important patrimoine. Il vise à demi-mot l'un des objectifs de l'article 39 du traité de Rome, repris dans feu le projet de traité établissant une constitution pour l'Europe, qui donne pour but à la Politique agricole commune "d'assurer un niveau de vie équitable à la population agricole, notamment par le relèvement du revenu individuel de ceux qui travaillent dans l'agriculture.» Il soutient que le revenu individuel ne doit pas être calculé en fonction du seul revenu courant des agriculteurs, mais en prenant également en compte le montant de leur patrimoine. Le calcul d'un statut économique des exploitants agricoles permettrait alors de mieux connaitre la situation économique des ménages agricoles et, à terme, de repenser la politique agricole en tenant compte des profits patrimoniaux des agriculteurs; les différences entre pays de l'Union européenne étant sur ce point importantes et significatives.

5 Jean-Pierre Butault, Nathalie Delame et Philippe Lerouvillois examinent dans leur article, les questions de la formation et des inégalités de revenu des ménages agricoles. Contrairement à l'article de Berkeley Hill qui utilise différentes expériences nationales (États-Unis, Danemark, France et Royaume-Uni) pour illustrer son propos, ces trois auteurs examinent uniquement le cas français. Leur article montre que les disparités de revenu global des ménages sont moins importantes que celles de revenu agricole. Ces auteurs considèrent alors que les salaires obtenus par l'un des membres du ménage dans un autre secteur d'activité pallient les déficiences structurelles d'un certain nombre d'exploitations. Ils esquissent implicitement une autre raison des mutations de la famille agricole. C'est aussi parce que les prix des biens agricoles sont insuffisants et la structure 
des exploitations non optimale que les ménages agricoles ont tendance à diversifier leurs sources de revenu et à modifier la répartition des tâches entre membres du ménage.

Stéphane Krebs prolonge cette analyse en soutenant, sur la base d'une analyse statistique d'un panel de 1800 exploitations individuelles françaises, que le choix de la pluriactivité est, en fait, un moyen d'assainir la situation financière de l'exploitation. En facilitant l'accès au crédit bancaire, la pluriactivité dégage des marges de manœuvre financières et favorise l'investissement.

7 Reste le problème de la transmission. À un horizon plus lointain, la pérennité des exploitations ne passe pas uniquement par un niveau de revenu satisfaisant. Elle exige aussi qu'au moment de la cessation d'activité, il y ait un repreneur. De nombreuses communications ont rappelé à cette occasion qu'en agriculture la transmission parentenfant reste le modèle. L'incapacité des pouvoirs publics à réaliser l'objectif «une installation pour un départ » traduit, cependant, les mutations en cours et le désintérêt de nombreux enfants d'agriculteurs pour l'exploitation familiale. La faiblesse des revenus dégagés par l'exploitation est sans doute une cause de ce désintérêt. L'article de Rob Burton, Heike Fischer et Gerald Schwartz du Macaulay Institute en Écosse traite de ces changements dans les modèles de succession en Ecosse et de leurs conséquences sur les politiques de développement local. Une exploitation en moins, c'est une source de lien social, d'échange et de production en moins. L'activité économique peut alors logiquement s'en ressentir.

8 Tous ces articles ont fait l'objet d'une relecture minutieuse du Comité de Rédaction de la revue et du Comité éditorial constitué à cette occasion. Les organisateurs du colloque et coordonnateurs de ce numéro remercient plus particulièrement Catherine Benjamin, Philippe Perrier-Cornet et Michel Blanc pour ce travail de suivi éditorial sans lequel ce numéro n'aurait pas pu être constitué. Ils sont également reconnaissants aux orateurs du colloque et auteurs des articles de ce numéro d'avoir enrichi les débats.

9 Il est désormais entre les mains des lecteurs qui sont les seuls à pouvoir décider in fine si le travail effectué sera fécond, autrement dit source de débats, de controverses et de nouvelles connaissances.

\section{AUTEURS}

\section{FRANCE CAILLAVET}

INRA, Ivry-sur-Seine

FRANÇOIS FACCHINI

Université de Reims

CATHERINE MOREDDU

OCDE 